\title{
An Improved Transformer for LLC Resonant Inverter for Induction Heating Applications
}

\author{
Amira ZOUAOUI- \\ KHEMAKHEM \\ Laboratoire Systèmes Electriques \\ (L.S.E.-LR11ES15) \\ Ecole Nationale d'Ingénieurs de \\ Tunis, Université Tunis El Manar \\ BP.37-1002-Tunis le Belvédère, \\ Tunisie
}

\author{
Hamed belloumi \\ Laboratoire Systèmes Electriques \\ (L.S.E.-LR11ES15) \\ Ecole Nationale d'Ingénieurs de \\ Tunis, Université Tunis El Manar \\ BP.37-1002-Tunis le Belvédère, \\ Tunisie
}

\author{
Ferid KOURDA \\ Laboratoire Systèmes Electriques \\ (L.S.E.-LR11ES15) \\ Ecole Nationale d'Ingénieurs de \\ Tunis, Université Tunis El Manar \\ BP.37-1002-Tunis le Belvédère, \\ Tunisie
}

\begin{abstract}
A new trend in power converters is to design a planar transformer that aims for low profile. However, at high frequency, the planar transformer $\mathrm{AC}$ losses become significant due to the proximity and skin effects. In this paper the most important factors for planar transformer (PT) design including winding loss, core loss, leakage inductance, and stray capacitance have individually been investigated. The tradeoffs among these factors have to be analysed to achieve optimal parameters. We will show a strategy to reduce losses in the primary coil of the transformer. The loss analysis of th PT was verified by the Finite Element Method (FEM) Simulations (ANSOFT MAXWELL FIELD SIMULATOR 2D) and could be utilized to optimize the transformer design procedure. Finally, the proposed PT has been integrated into an LLC Resonant Inverter for Induction Heating Application to test at high signal levels.
\end{abstract}

Keywords-planar transformer; induction heating; Finite Element Method; skin effect; diffusive representation

List of Symbols:

$\mathrm{b}$ : Width of the plate

$C$ : Compensation capacitor

$F_{0}$ : Resonant frequency

$\mathrm{h}$ : Height of the plate

I : Primary current

$I_{S}:$ Secondary current

$\mathrm{K}$ : Coefficient of additional losses

$L_{s}:$ Secondary inductance

$L$ : Load inductance

$\mathrm{m}$ : Number of layers

$N$ : Primary turns of the transformer

$\mathrm{p}$ : Diameter of the cross section of the conductor

$P$ : Power

$R$ : Load resistance

$R_{a c}:$ AC resistance of the winding

$R_{d c}$ : DC resistance of the winding

$\mathrm{S}:$ Area of the cross section of the conductor

$S_{r e c}:$ Rectangular section

$V_{\text {in }}$ : Fundamental voltage

$V_{b}$ : Primary voltage

$\Phi$ : Phase shift between fundamental voltage and current

$\delta$ : Skin depth $\rho:$ Copper resistivity

$\xi$ : Normalized value of the diameter of a strand

$\mu$ : Permeability

\section{INTRODUCTION}

High-frequency power transformers (HFPT) are largely used in many electrical and electronic designs. Switching at higher frequencies is an interesting option to reduce passive components and isolation transformers. They allow adapt different levels of voltage and provide electrical and galvanic isolation between both windings. However, there is not a standardized procedure for design transformers, and every manufacturer keeps confidential his method. Optimized design can save power, volume, weight and money for the manufacturer and for the customer. Most of researchers studied the theory of HFPT and its usage in power systems.

Due to eddy current effects, winding losses in highfrequency magnetic components are appreciably greater than those Calculated from the dc resistance of the winding. These losses must then be computed from the AC resistance of the winding which includes the DC resistance and a factor of excess loss related to skin and proximity effects [1].

We have shown in [2] two options to reduce highfrequency copper losses without significantly increasing the space occupied by these connections. Both options rely on the parallelization of copper plates. In the first, plates are interchanged to equalize the flux experienced by them. In the second, plates are interleaved to decrease the magnetic energy around the conductor.

PCB implementation of planar windings replicates the structure of conventional litz wires to reduce the AC losses. This structure consists of a set of fine trace electrically connected in parallel which transposed using changes of both trajectory and layer. An appropriate transposition strategy is essential to reduce the winding AC losses. However PCBs with a high number of vias could result expensive and not reliable [3].

A quick and simple method derived for obtaining the $\mathrm{AC}$ leakage impedance of a transformer, from the calculated values of DC winding resistance and DC leakage inductance. 
It is rather unfortunate that calculating the DC leakageinductance components accurately is a fairly lengthy process, although it mainly consists of substituting numbers in the given formulas [4].

[5] Explained a new method for core selection, based on the estimation of current density. The final algorithm presented allows to choice the magnetic core quicker, and designs a more efficient device. Furthermore, the method allows design a transformer with a broad range of frequency.

An improved high-frequency magnetic-integrated planar transformer was demonstrated in [6]. Compared with conventional planar integrated magnetic, the improved structure has better leakage inductance adjustment. The transformer was fabricated with two common EE shape magnetic cores along with magnetic insertions placed between the primary and secondary windings outside it. A threedimensional finite-element model is used to investigate the eddy current losses and the adjustment of the leakage inductance.

A compact planar transformer with an improved winding configuration has been designed and fabricated in [11]. The meander-type design was engraved over the surface of a planar ferrite core, so that the primary and secondary windings can fit into the engraved design. This structure, was covered with another ferrite core, thus forming a compact planar transformer. Apical type AV polyimide film of $0.025 \mathrm{~mm}$ thickness is used between the windings to prevent short circuit. A coupling coefficient of $>0.93$ achieved, while the primary and secondary inductance was $\sim 1600 \mathrm{nH}$. A coupling coefficient for air core transformer with the windings of identical geometry was $>0.35$ and the inductance of the windings near $160 \mathrm{nH}$. Fabricated transformer prototype has significantly a higher coupling coefficient and inductance of the windings in comparison with the previously developed planar transformer of the similar design.

In this paper, an HFPT for induction heating device is presented, thus its losses analysis. To achieve our HFPT, The choice and sizing of the primary and secondary of the transformer are necessary. Hence a transformer has been designed in order to adapt the voltage and current levels between the converter and the resonant tank.

An analytical method for the calculation of losses in HFPT followed by a simulation is perfomed. The simulation results show the good performance of proposed system and verify the mathematical analysis. Additionally, this new topology provides higher power rating on utility voltage at the load side.

\section{OPERATION OF THE PROPOSED INVERTER}

Fig. 1 illustrates the power circuit of proposed induction heating diagram that employs an $L L C$ resonant inverter configuration for induction-heating applications. The inverter consists of two switches with antiparallel diodes, a compensation capacitor (C), a series inductor (Ls), and an induction coil that comprises a series combination of a resistor $(R e q)$ and an induction coil inductor ( Lcoil).

You need to use half bridge capacitor inverter that eliminates the DC component and ensures a zero average current, and voltage in the primary of the transformer.

If you want to work at full power, you only have to operate the two half bridge, otherwise the half-bridge is sufficient for a power of $5 \mathrm{~kW}$.

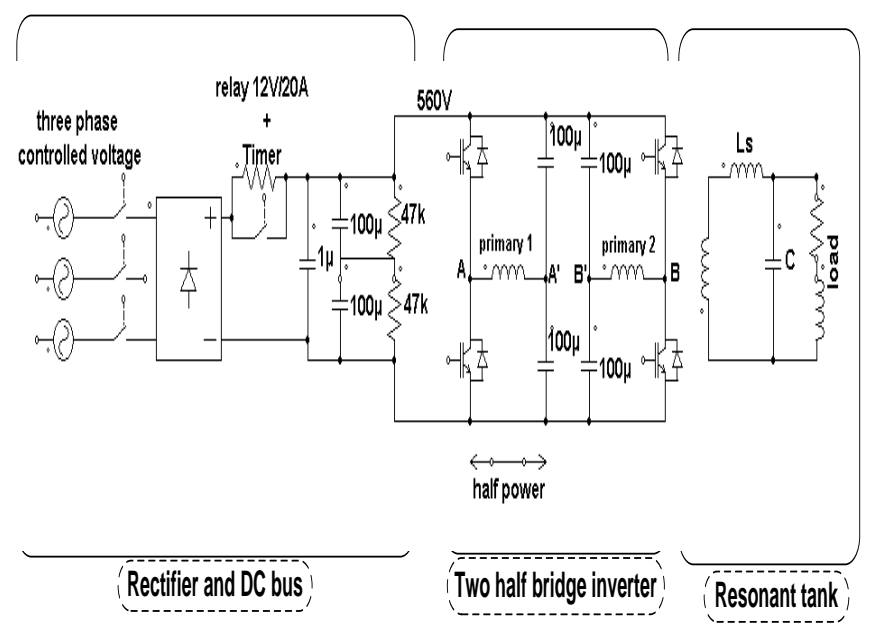

Fig. 1. Electrical diagram of the induction heating system

To ensure the compensation of the reactive, the commands must coincide with the zero crossing of the current Ic in the compensation capacity $\mathrm{C}$.

Fig. 2 displays the current conduction through the inverter during different modes of the power transfer cycle.

During Mode 1, a positive voltage is applied to the gate of the high active power MOSFET. As a consequence, high MOSFET is turned-on. mosfet is conducting and transfer power to the resonant tank through the coupling transformer in our circuit. In Mode 2, the mosfets are transitioning, and the upper mosfet turns off slightly before the bottom one turns on. Here, current is conducted through the free-wheeling diode of the lower mosfet. In Mode 3, the lower mosfet turns on, and the resonant tank throws the power back through mosfet. In Mode 3, both mosfets are off during the transition, and the upper mosfet's free-wheeling diode conducts the current.

\section{LOSSES ANALYSIS OF HIGH FREQUENCY PLANAR TRANSFORMER}

When power converters operate at high frequency, the design difficulty for the transformer becomes much higher. The current density redistribution inside the winding wires (skin and proximity effects) strongly increases the copper losses. 


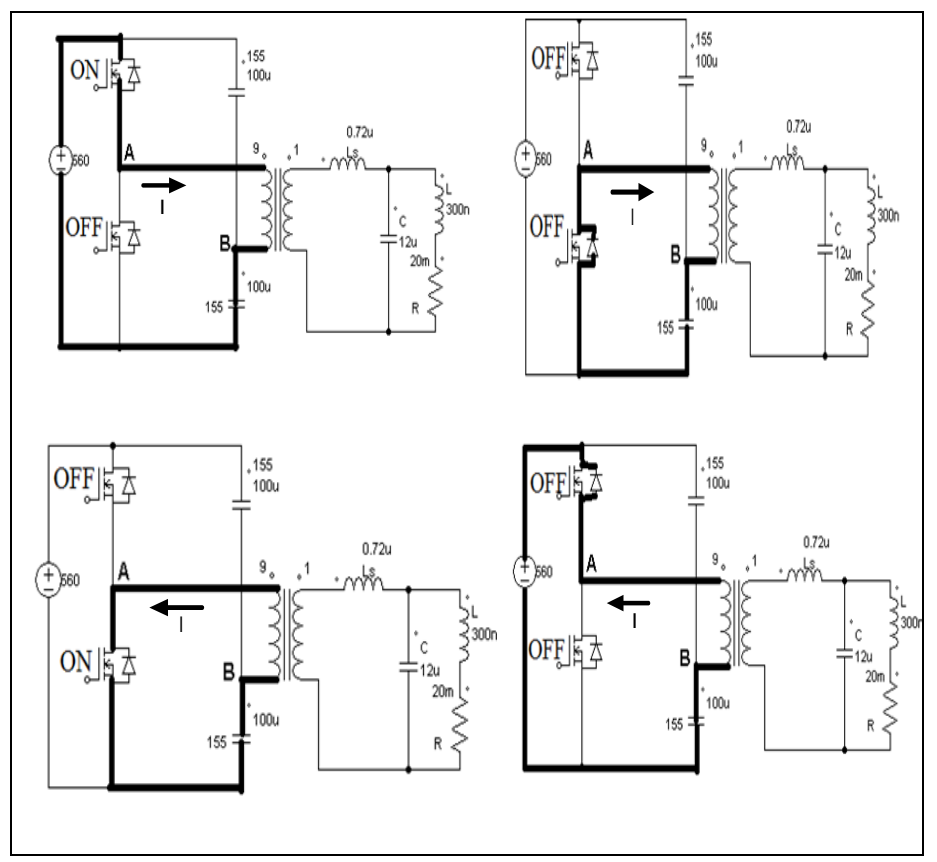

Fig. 2. Analysis of the inverter

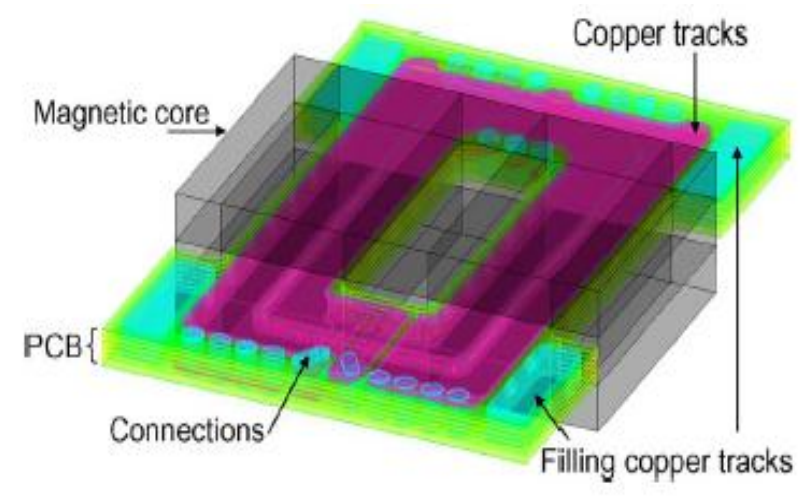

Fig. 3. Planar transformer

A. Choice and sizing of the primary of the transformer

Determining the number of primary turns:

Installation must be given to the resonance frequency defined by:

Resonance frequency

$$
F_{0}=\frac{1}{2 \pi \sqrt{(L S / / L) C}}=100 \mathrm{khz}
$$

It sets the $\Phi$ switching angle $20^{\circ}$

Data: $\mathrm{V}_{\mathrm{b}}=560 \mathrm{~V} ; \mathrm{Vin}=504 \mathrm{~V} ; \mathrm{L}=0.3 \mu \mathrm{H} ; \mathrm{R}=20 \mathrm{~m} \Omega$; $\mathrm{P}=10 \mathrm{~kW}$

The 14 turns will be divided over 7 epoxy plates as follows:

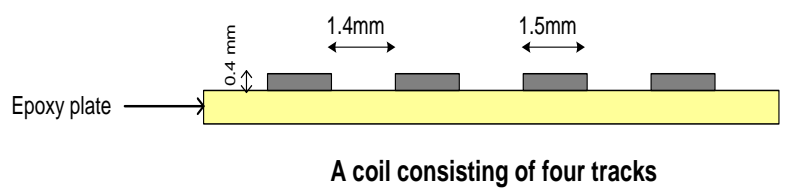

The thickness of the planar turn has to be two times the skin depth.

For $100 \mathrm{kHz}: \quad \delta=209 \mu \mathrm{m} ; \quad \rho=\frac{1}{5810^{6}}$

$R_{\text {coil } D C}=\rho \frac{L}{S}=0.028 \Omega$;

$R_{\text {total }}=0.0072 \Omega$;

For a length of $100 \mathrm{~mm}: R_{\text {total }}=0.00072 \Omega$

Coppers losses in a single turn in DC are given by:

$$
P_{c u}=R_{\text {total }} * I^{2}=0.317 \mathrm{~W}
$$

Copper primary losses roundtrip in DC mode:

$$
P_{\text {copper }} * 14 * 2=8.89 \mathrm{~W}
$$

Now to calculate the AC losses in a track

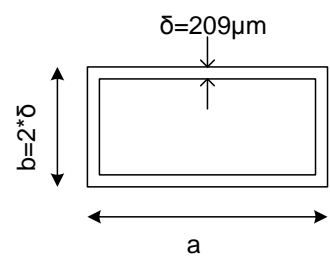

$$
\begin{aligned}
S_{A C}=S_{\text {ext }}-S_{\text {int }} & =(a * b)-[(a-2 \delta)(b-2 \delta)] \\
& =2 \delta(a+b-2 \delta)
\end{aligned}
$$

Or $\mathrm{b}=2 \delta$

$$
S_{A C}=2 \delta * a=2 * 0.209 * 1.5=0.6 \mathrm{~mm}^{2}
$$

We can therefore deduce that

$$
S_{A C}=S_{D C} \text { and } P_{\text {copper } A C}=P_{\text {copper } D C}
$$

In the high frequency, the fact of increasing the thickness of a conductor does not reduce its apparent resistance, since the current density is confined in a skin thickness. A second solution to reduce losses is to connect two conductors in parallel.

In practice, the current density will be distributed as if both drivers were a single thicker conductor, so it must wait no significant improvement from the standpoint of losses.

The current distribution in the tracks is not uniform, and this is owed to the skin effect, the current is concentrated in the extremities. We note that when conductors are side-by side, the high concentration of the flux in the region causes high current densities in this small part of the conductor and, as a consequence, high copper losses. 


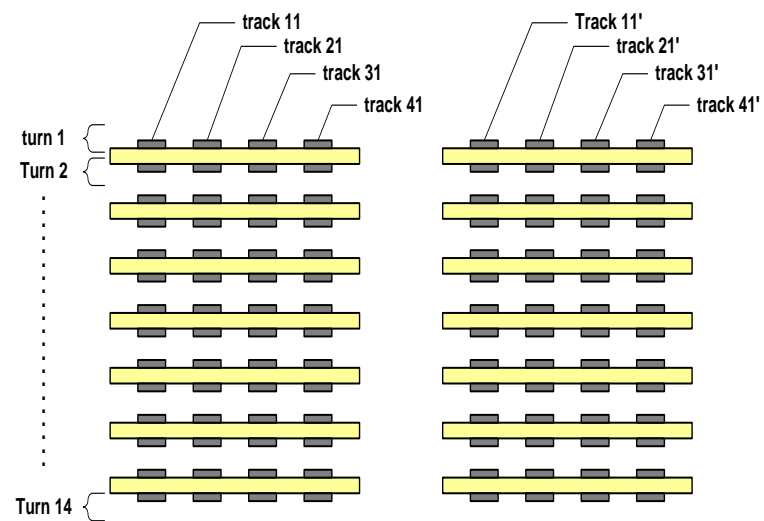

Fig. 4. 14 primary turns

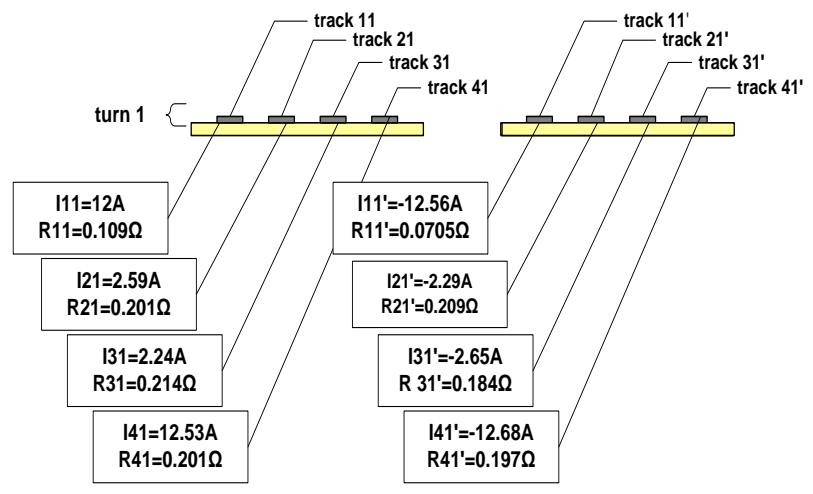

Fig. 5. Current distribution in a track

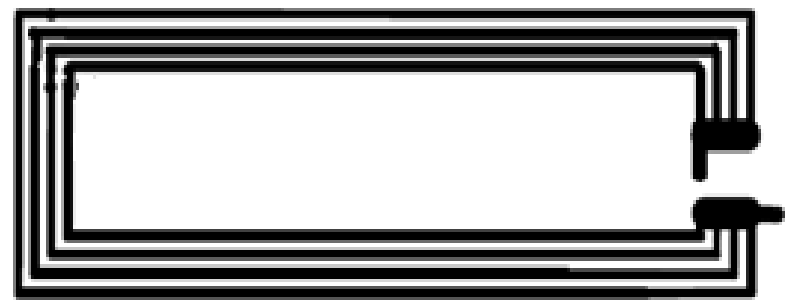

Fig. 6. 1 turn with 4 tracks

To mitigate skin effect and excessive losses due to the large rectangular tracks, another solution proposed by [7] is to use the planar Litz wire. Inspired by the Litz round wire, Litz planar structure has been proposed to reduce the strength of the alternative regime of a planar conductor.

It proposes a new form of planar litz wire. The proposed idea is to translate the turns with each other to force the current to pass around the turns and not be limited to the edges of the rectangular turns. The proposed solution is to divide each turn in four small tracks which leave together in parallel to form the required number of turns, then perform an intersection between the four tracks of each turn.

Resistance in each of the turns, deducted by finite element method (FEM), is nearest value. But we note that the resistance decreases significantly at the 14 turn. This can be explained by the proximity of the turn of the secondary winding traversed by a high current (fig.7).

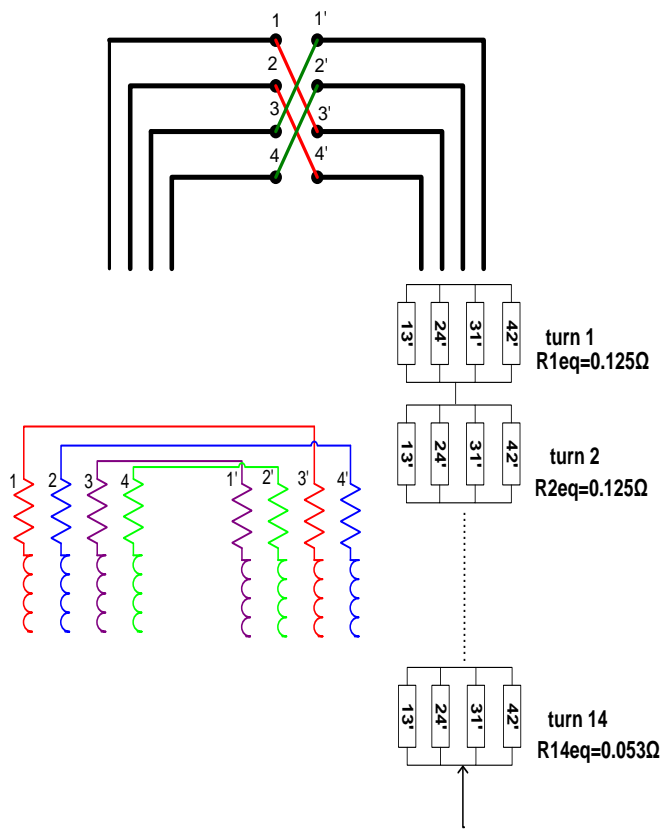

Fig. 7. Distribution of primary turns

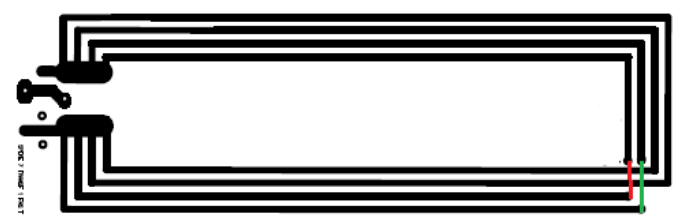

Fig. 8. Design of a new turn on ISIS

\section{B. The secondary of the transformer}

The shape of the secondary single-turn essentially depends on the ferrite constituting the transformer. In fact the latter will consist of six blocks of ferrites.

We must be adapted a solution to cool it when the shape and dimensions of the single-coil are fixed.

The idea is simple; it is to design an internal channel that will serve for the flow of coolant. The section of this channel must be maximum; however it must not interfere with the feasibility of the mono-spiral. For this we set the wall thickness to at least $3 \mathrm{~mm}$. To optimize the shape of the single turn an analytical calculation followed by a simulation by the finite element method (FEM) was conducted.

To determine the optimum dimensions of the canalization of the single turn, we compared the total losses Joules effect for the different forms section.

\section{AnAlytical Method FOR the CAlCUlation OF LOSSES}

Winding losses in transformers increase dramatically with high frequency due to eddy current effects. Eddy current losses, including skin- and proximity-effect losses, seriously impair the performance of transformers in high-frequency power conversion applications. Both the skin effect and the proximity effect cause the current density to be non-uniformly 
distributed in the cross section of the conductor and thus cause a higher winding resistance at higher frequency.
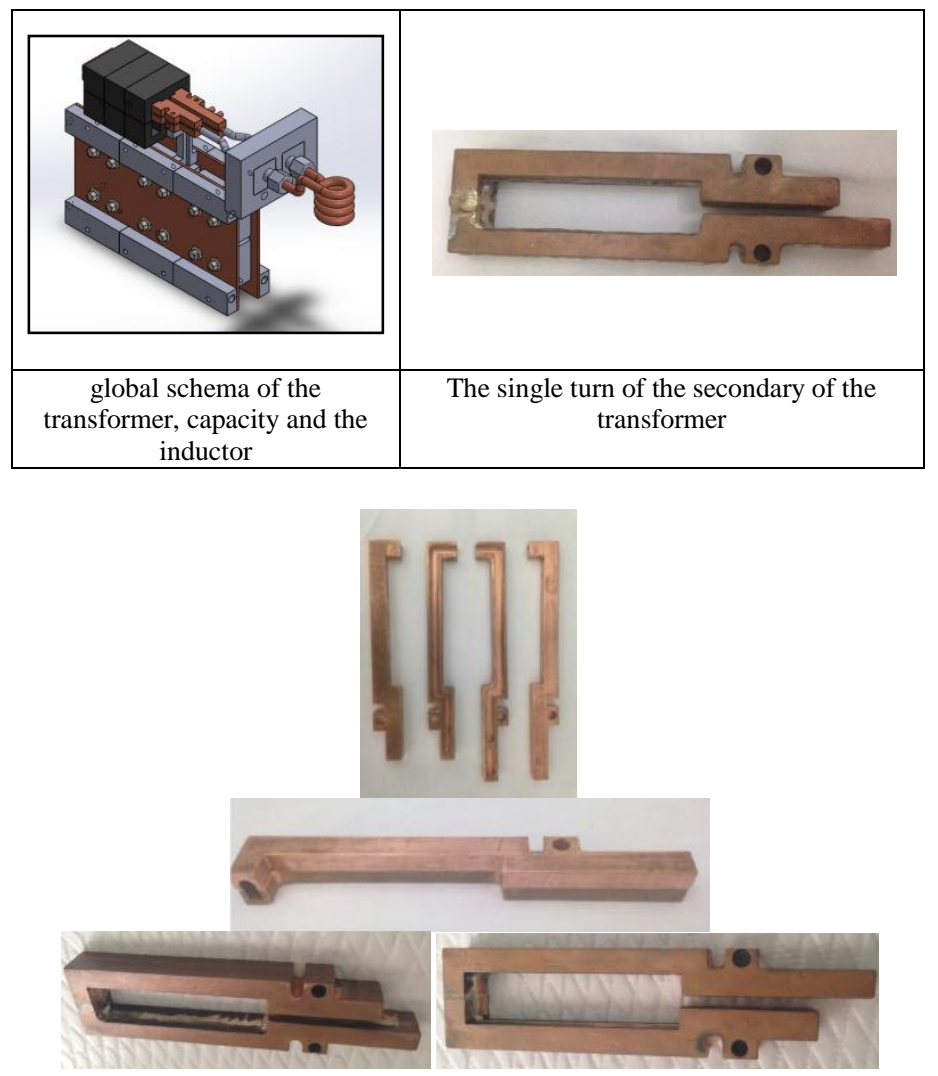

Fig. 9. The single turn of the secondary of the transformer

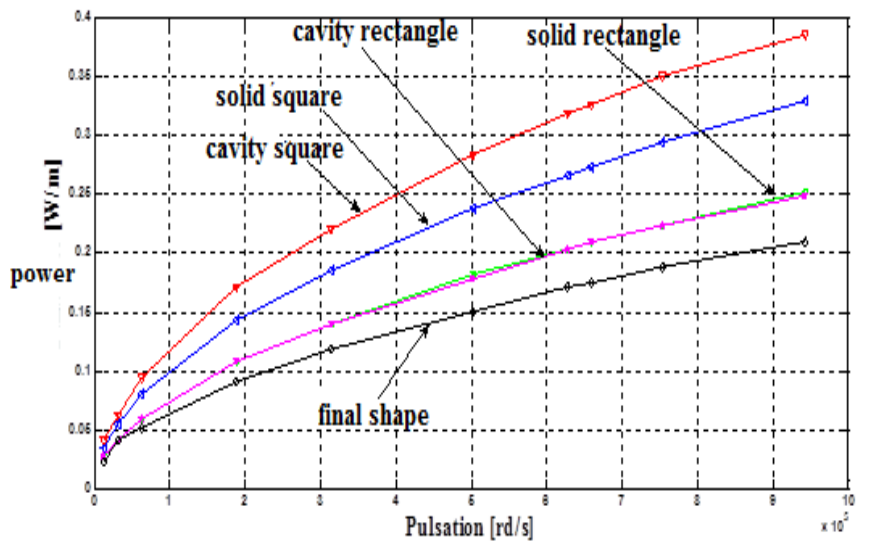

Fig. 10. Justification for the choice of the form of canalization of the single turn

The most commonly used equation that characterizes winding losses is Dowell's equation [4]-[8]

$$
\begin{gathered}
R_{a c, m}=R_{d c, m} \frac{\xi}{2}\left[\frac{\sinh \xi+\sin \xi}{\cosh \xi-\cos \xi}+(2 m-1)^{2} \frac{\sinh \xi-\sin \xi}{\cosh \xi+\cos \xi}\right] \\
\text { With } \xi=\frac{\sqrt{\pi}}{2} \frac{d}{\delta}
\end{gathered}
$$

\section{A. Empirical formula}

Circular conductor forms:
For this particular form, the calculations are less complex and more accurate results.

The only parameter which is generally considered is the ratio $\mathrm{K}=\frac{R_{a c}}{R_{d c}}$ which indicates a form of inadequate conductor when its value deviates too much from the unit.

Several empirical formulas have been proposed; that of Levasseur [9] is particularly simple and leads to errors of less than $2 \%$ :

$$
\begin{gathered}
\mathrm{K}=\sqrt[6]{\frac{3}{4}^{6}+\left(\frac{S}{p \delta}\right)^{6}}+0.25 \\
R_{a c}=R_{d c} * \sqrt[6]{{\frac{3^{6}}{4}}^{6}+\left(\frac{S}{p \delta}\right)^{6}}+0.25
\end{gathered}
$$

Rectangular form of the Conductor:

In this case, the calculations are much more complex and remain unclear because of the assumptions used about the distribution of the magnetic field; authors who have tried these calculations have often completed their work with delicate experiments.

According to estimates [10], a coefficient of 1.15 seems most probable correct errors due to non-compliance of the distribution of the field.

$$
R_{a c}=1.15 *\left(R_{d c} * \sqrt[6]{\frac{3^{6}}{4}+\left(\frac{S}{p \delta}\right)^{6}}+0.25\right)
$$

\begin{tabular}{|c|c|c|c|c|c|c|}
\hline Frequency & \multicolumn{2}{|c|}{$1 \mathrm{kHz}$} & \multicolumn{2}{|c|}{$10 \mathrm{kHz}$} & \multicolumn{2}{|c|}{$100 \mathrm{kHz}$} \\
\hline $\begin{array}{l}\text { losses calculated by } \\
\text { the corrected } \\
\text { empirical formula }\end{array}$ & $\begin{array}{l}23 \\
W\end{array}$ & $5 \%$ & $66 \mathrm{~W}$ & $6 \%$ & $204 \mathrm{~W}$ & $9 \%$ \\
\hline $\begin{array}{l}\text { Losses calculated by } \\
\text { (FEM) }\end{array}$ & \multicolumn{2}{|c|}{$22 \mathrm{~W}$} & \multicolumn{2}{|l|}{$63 \mathrm{~W}$} & \multicolumn{2}{|l|}{$227 \mathrm{~W}$} \\
\hline
\end{tabular}

TABLE I. COMPARATIVE TABLE OF LOSSES CALCUlATED By DifFERENT METHODS

\begin{tabular}{|l|l|l|}
\hline $\begin{array}{l}\text { Losses calculated by the } \\
\text { empirical formula }\end{array}$ & $\begin{array}{l}\text { losses calculated by } \\
\text { the corrected empirical } \\
\text { formula }\end{array}$ & $\begin{array}{l}\text { Losses calculated by } \\
\text { (FEM) }\end{array}$ \\
\hline $177 \mathrm{~W}$ & $204 \mathrm{~W}$ & $227 \mathrm{~W}$ \\
\hline Error $21 \%$ & Error $9 \%$ & \\
\hline
\end{tabular}

As the frequency increases, the error increases.

\section{B. Bessel Formula}

The conductor is carrying a current $I_{S}(\mathrm{rms})=304 \mathrm{~A}$

The complex power due to the skin effect is given by:

$$
\begin{aligned}
& P_{\text {skin }}=\frac{\sqrt{j w \mu \sigma}}{2 \pi r \sigma} \frac{I_{0}(r \sqrt{j w \mu \sigma})}{I_{1}(r \sqrt{j w \mu \sigma}} I I^{*} \\
& R=\text { Real } \frac{\sqrt{j w \mu \sigma}}{2 \pi r \sigma} \frac{I_{0}(r \sqrt{j w \mu \sigma})}{I_{1}(r \sqrt{j w \mu \sigma}}
\end{aligned}
$$

With: $I_{0}(r \sqrt{j w \mu \sigma})$ et $I_{1}(r \sqrt{j w \mu \sigma})$ are modified Bessel functions of the first kind of order zero and one.

r: radius of a circle

$\sigma:$ resistivity

In the case of a single rectangular conductor, we first treated the rectangular shape to an equivalent circle keeping the same section. 


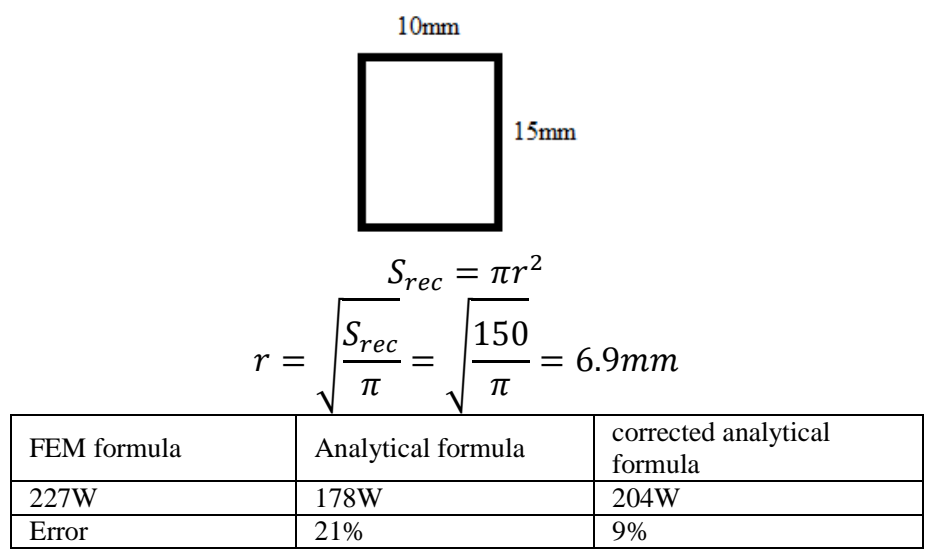

C. Calculating the internal impedance owed to the skin effect and proximity of a rectangular conductor

$$
\begin{aligned}
& R_{\text {recP }}=\frac{1}{\sigma b \delta} * \frac{\sinh \left(\frac{2 h}{\delta}\right)+\sin \left(\frac{2 h}{\delta}\right)}{\cosh \left(\frac{2 h}{\delta}\right)-\cos \left(\frac{2 h}{\delta}\right)} \\
& L_{r e c P}=\frac{1}{\delta \omega \sigma b} \frac{\sinh \left(\frac{2 h}{\delta}\right)-\sin \left(\frac{2 h}{\delta}\right)}{\cosh \left(\frac{2 h}{\delta}\right)-\cos \left(\frac{2 h}{\delta}\right)}
\end{aligned}
$$

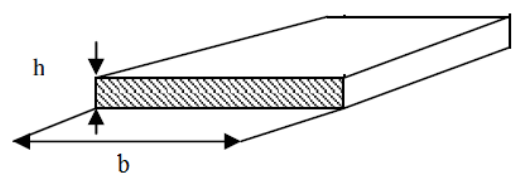
plate

With b: width of the plate

h: height of the

Knowing that the width should be strictly greater than the height that this formula is true so we take our case $b=19 \mathrm{~mm}$ and $\mathrm{h}=12 \mathrm{~mm}$.

TABLE II. Comparison Between Fem And the Analytical Method

\begin{tabular}{|l|l|l|}
\hline & FEM & Analytical \\
\hline Losses & 461 & 406 \\
\hline Resistance & 0.0049 & 0.0043 \\
\hline Inductance & $2.135^{\mathrm{E}-007}$ & $6.9109 \mathrm{e}-009$ \\
\hline
\end{tabular}

The error between the two powers is $11 \%$.

We compared the analytical expressions obtained from different methods. In fact the first case (empirical calculation) has an acceptable convergence of results by finite element method (FEM) and analytical losses. This convergence is only true when adding a correction factor of 1.15. This coefficient is valid for the second case too which replaces a rectangular conductor with a circular conductor with the same cross section and the error is minimal. As can be seen, the theoretical loss calculations are in agreement with the results obtained with the Finite Element simulations. As the two dimensional finite element analysis allows the estimating of the fringing effect at the end of the conductors, the simulated losses may exceed the calculated transformer losses a little.

To study the internal current constraint in our transformer during operation, we have been led to make a digital model.

Achieving diffusive admittance was identified. Diffusive symbol approached is shown in Fig. 11.

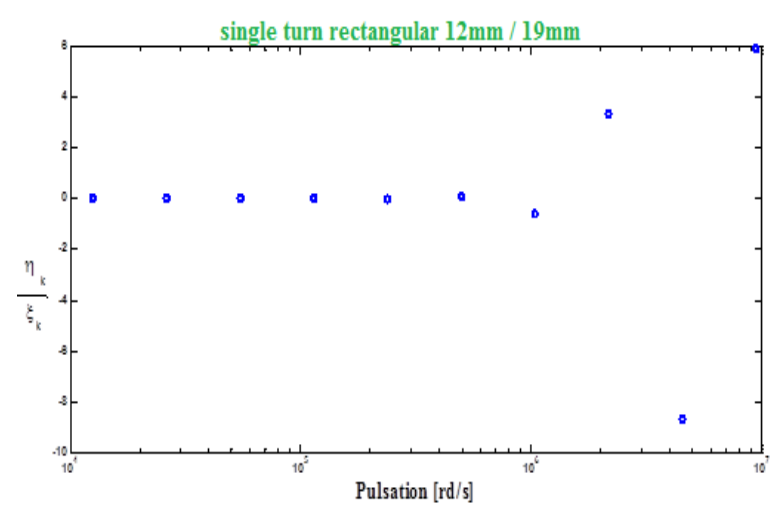

Fig. 11. Diffusive approached symbol of admittance $\frac{H(P)}{l}$
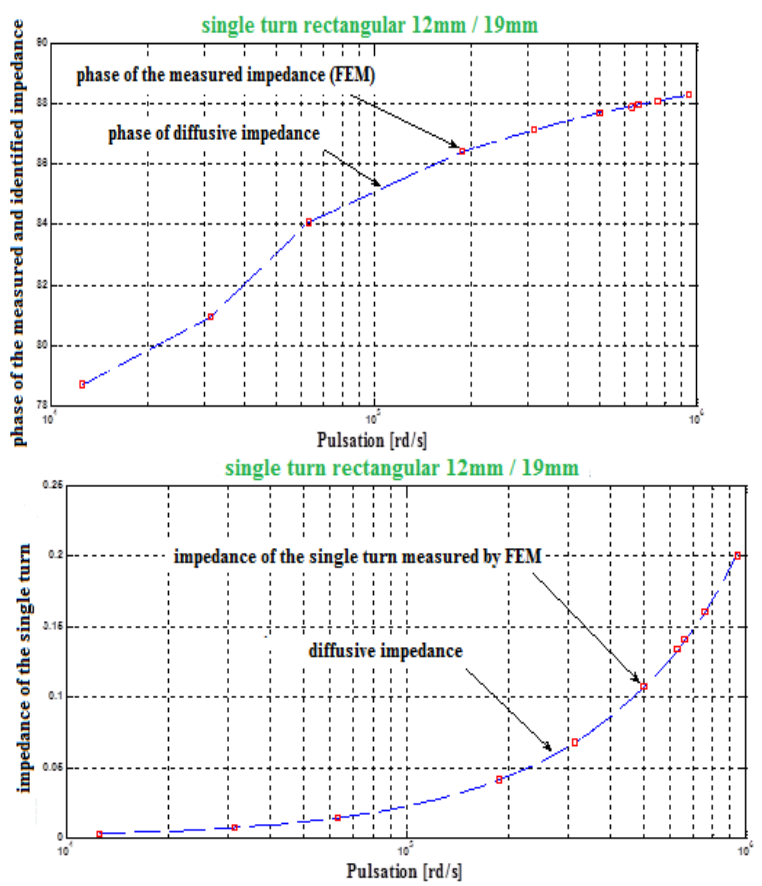

Fig. 12. Comparison of the estimated admittance by FEM and identified

In Fig.12, the frequency response of the admittance thus identified is compared to the response obtained using the parameters determined by FEM simulation. The agreement is quite satisfactory.

\section{SimUlation RESUlts}

To validate the loss estimation mentioned above, the detailed losses in a high frequency planar transformer are studied using the Finite Element Method(FEM) in detail.

Finite Element Method (FEM) can be used for calculating transformer characteristics and stray parameters.

2-D simulations perform an analysis on the transformer based on its symmetry axes.

Determining losses by Joule effect is using the Maxwell computer through the formula of losses:

$$
P=\frac{1}{2 \sigma} \int_{V} J \cdot J^{*} d V
$$


To realize the finite element modeling, we impose for the primary winding a current of 30A peak for the four parallel strands that form our proposed Litz wire, and the secondary we impose a current of $432 \mathrm{~A}$ peak for the single turn. This finite element modeling of the copper losses was performed at a frequency of $100 \mathrm{kHz}$.

The parameters of the transformer studied are shown in Fig 13. For this planar transformer, the red plates on the top in the model represent the 14 primary turns and the red one in the other side of the transformer represent the secondary turn.

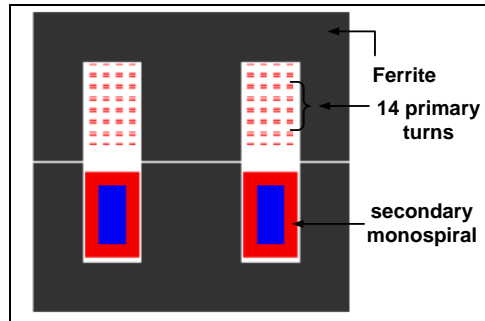

Core size: EE 7032 A

Material: ferrite

Primary turns: 14

Secondary turns: 1

Effective core: $2049 \mathrm{~mm}^{2}$

Fig. 13. Shows the magnetic flux distribution in frontal model of the planar transformer

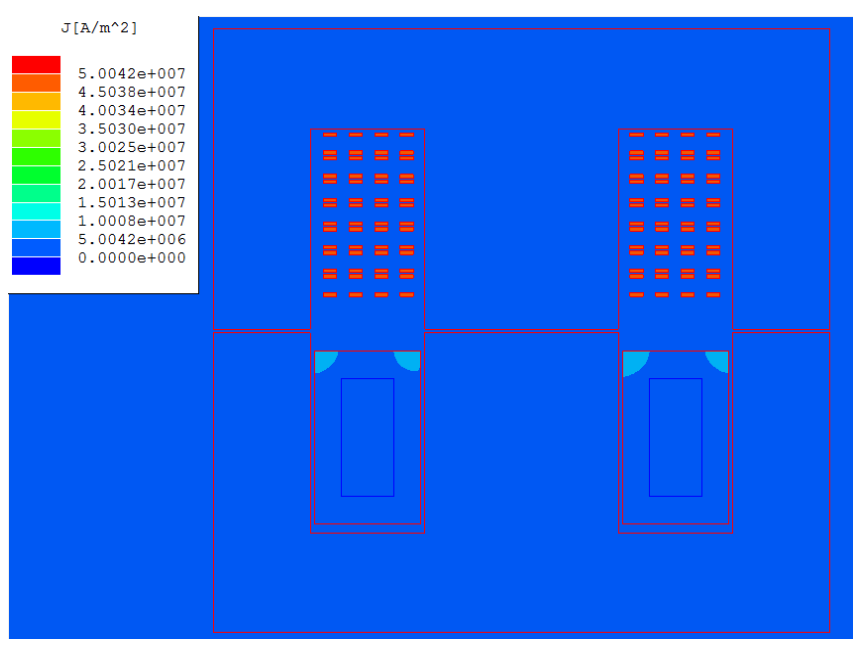

Fig. 14. FEM simulation at $\mathrm{F}=100 \mathrm{~Hz}$

For each frequency of $100 \mathrm{~Hz}$ and $100 \mathrm{kHz}$, we simulate the contour plot of current density.

The loss estimation takes account of switching-type waveforms encountered in power supplies, inclusive of high frequency skin and proximity effects in windings.

This planar transformer is used in a half bridge converter and its associated voltage and current waveforms are shown in Fig.16. The voltage across the primary winding is a square wave with peak values of-560/2 $\mathrm{V}$ and $560 / 2 \mathrm{~V}$. The current through the primary winding is also a square wave with peak values of $-38 \mathrm{~A}$ and $38 \mathrm{~A}$.

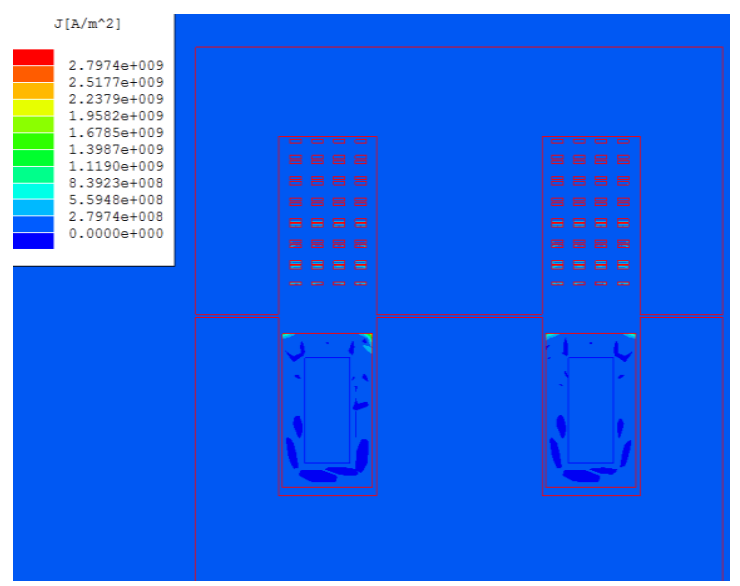

Fig. 15. FEM simulation at $\mathrm{F}=100 \mathrm{kHz}$

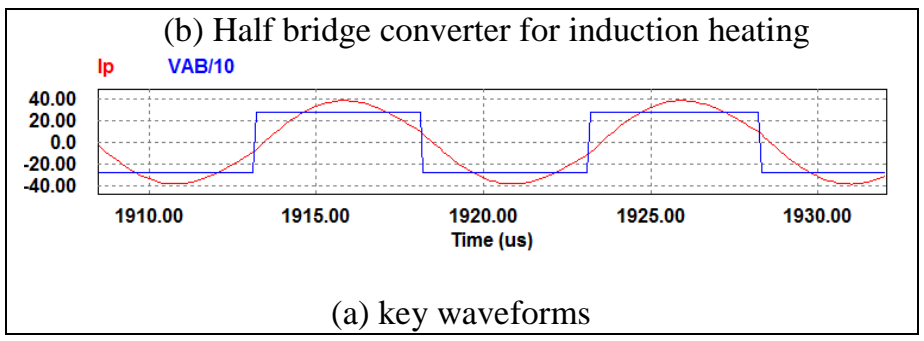

Fig. 16. The half bridge converter

\section{EXPERIMENTAL RESULTS}

The experimental results presented are for a $5 \mathrm{~kW}$ furnace.

Fig. 16 displays the voltage waveforms $V_{D S}$, of the high MOS and the resonant current. When the MOS is turned-on, the current is initially negative, evidence that circulates in the internal diode of the high MOS. Meanwhile, the MOS is turned-on at zero voltage. The current becomes positive until the order of his blocking, reducing the voltage $V_{a b}=+E$ between its terminals with no overvoltage thanks to CALCS. The test was carried under an input voltage $\mathrm{E}=400 \mathrm{~V}$, and a current IpM = 12A.

The temperature of the work piece is measured by an optical pyrometer and the Curie temperature at $875^{\circ} \mathrm{C}$ is achieved as desired.

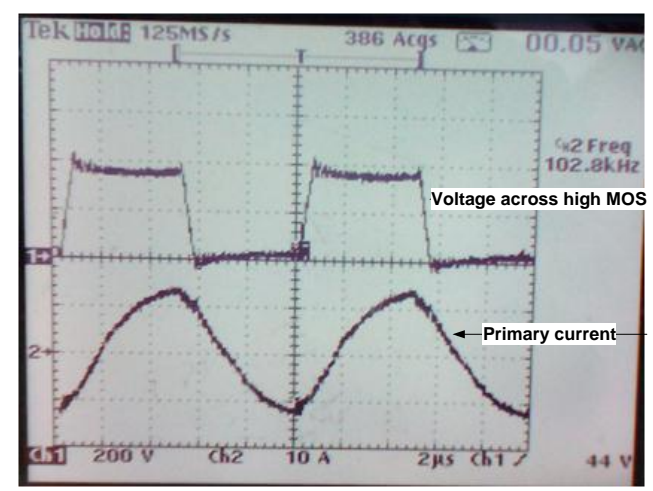

Fig. 17. Diagram viewing the output voltage of the MOS high and the primary current 
As a conclusion, these waveforms match up with the theoretical expected ones, and verify the proper operation of the proposed converter. Fig. 18 shows a photo of the implemented hardware prototype.

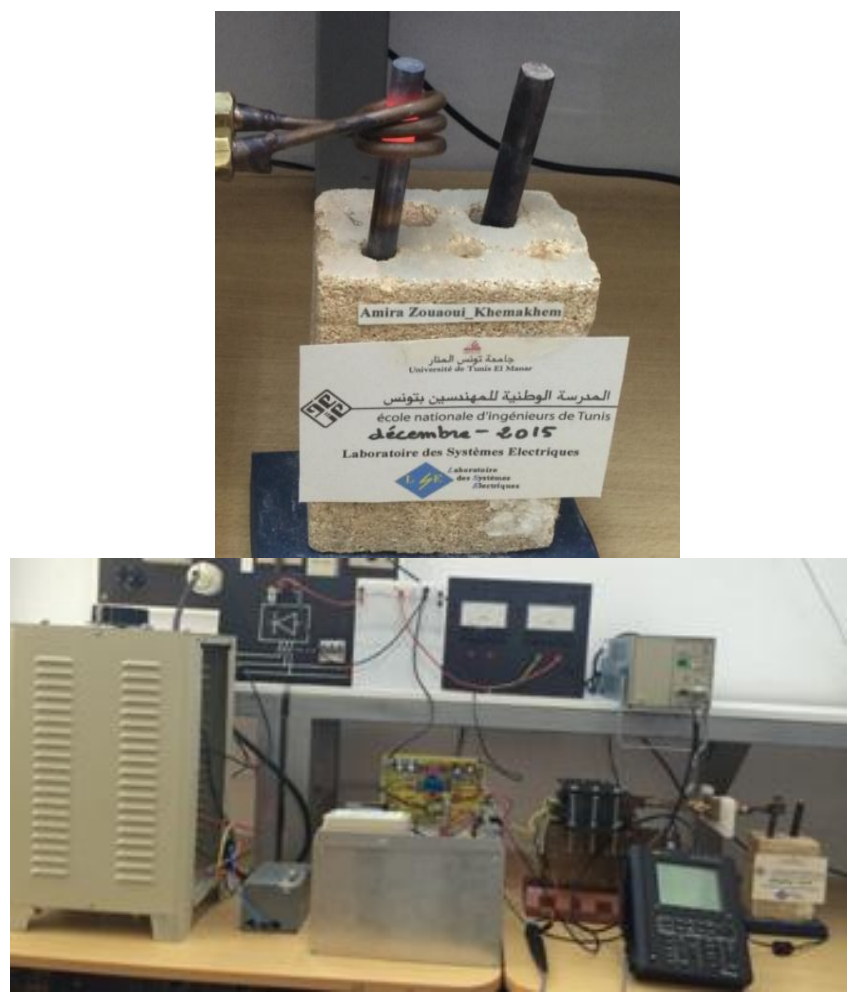

Fig. 18. experimental model

\section{CONCLUSION}

Planar transformers are more and more used in electronic power structures since they have major interests in congestion, performance and industrial production method. In the first part of this paper, we have sought to reduce the joule losses planar transformer due to the skin effect and proximity effect.

We have presented all the steps for building a highfrequency transformer model. Some parameters such as resistance and inductance of the single turn are affected by the variation of the frequency (the effects of skin and proximity) as well as the variation of the shape of the conductor. Such a phenomenon can model by adopting the diffusive representation. The different model parameters are estimated by the finite element method (FEM). It has been shown that it is possible to use with certain circumstances analytical methods to provide an order of magnitude of some parameters.
A 5-kW prototype has been designed and implemented to validate the analytical and simulation results. The experimental measurements validate the feasibility of the proposed converter.

\section{ACKNOWLEDGMENT}

This work was supported by the Tunisian Ministry of Higher Education and Research under Grant LSE-ENITLR11ES15

\section{REFERENCES}

[1] F. Tourkhani, P. Viarouge « Accurate Analytical Model of Winding Losses in Round Litz Wire Windings », IEEE transactions on magnetics, vol. 37 , no. 1 , january 2001

[2] B. Cougo, J. W. Kolar, G. Ortiz, « Strategies to Reduce Copper Losses in Connections of Medium-Frequency High-Current Converters », 7th Annual Conference of the IEEE Industrial Electronics Society (IECON 2011), Melbourne, Australia, November 7-10, 2011.

[3] I. Lope, J. Acero, J. Serrano, C. Carretero, R. Alonso and J.M. Burdio, « Minimization of Vias in PCB Implementations of Planar Coils with LitzWire Structure », IEEE 2015

[4] P.L Dowell, «Effects Of Eddy Currents In Transformer Windings » Proc Iee Vol 113, NO 8, August 1966

[5] Xavier Duran Reus, Foo Javier Quiros And Daniel Montesinos Miracle, «Design Of A High Frequency Transformer For An Induction Heating System » SSD2014

[6] Wayne Water And Junwei Lu, «Improved High Frequency Planar Transformer For Line Level Control (LLC) Resonant Converters », IEEE Magnetics Lettters, Volume 4 (2013)

[7] B. Cougo, J. W. Kolar, G. Ortiz « Strategies to Reduce Copper Losses in Connections of Medium-Frequency High-Current Converters » Proceedings of the 37th Annual Conference of the IEEE Industrial Electronics Society (IECON 2011), Melbourne, Australia, November 7 $10,2011$.

[8] A.Ducluzaux, « Extra Losses Caused In High Current Conductors By Skin And Proximity Effects », Cahier Technique N 83, edition 1977

[9] K. Ben Smida M. Elleuch, «Analyse Des Effets De Peau Et De Proximite Sur L'impedance Interne D'un Enroulement Statorique »

[10] Alexander W. Barr , "Calculation of Frequency-Dependent Impedance for Conductors of Rectangular Cross Section» AMP Journal of Technology Vol. 1 November, 1991

[11] Djuric S.M, Stojanovic G.M. "A Compact Planar Transformer With an Improved Winding Configuration" , IEEE Transactions on Magnetics (Volume:50, Issue: 11 ) Nov. 2014

[12] Ignacio Lope, Claudio Carretero, Jesus Acero, Rafael Alonso, and Jose M. Burdio "Frequency-Dependent Resistance of Planar Coils in Printed Circuit Board With Litz Structure" IEEE transactions on magnetics, vol. 50, no. 12, december 2014

[13] Ignacio Lope, jesus Acero, Claudio Carretero, "Analysis and Optimization of the Efficiency of Induction Heating Applications with Litz-Wire Planar and Solenoidal Coils" IEEE transactions on power electronics, September 2015 